Radiologe $2011 \cdot 51: 654$

DOI 10.1007/s00117-011-2227-6

Online publiziert: 15. August 2011

(c) Springer-Verlag 2011

\author{
T. Helmberger ${ }^{1} \cdot$ C. Herold ${ }^{2}$ \\ ${ }^{1}$ Institut für Diagnostische und Interventionelle Radiologie, Neuroradiologie \\ und Nuklearmedizin, Städt. Klinikum München, Klinikum Bogenhausen, München \\ ${ }^{2}$ Univ.-Klinik für Radiodiagnostik, Allgemeines Krankenhaus, \\ Medizinische Universität Wien, Österreich
}

\title{
Update Leberbildgebung
}

Liebe Leserinnen und Leser,

für die Leber ein Update - wie langweilig, soviel gibt's doch da gar nicht - oder doch? Ist das letzte Themenheft zur Leberbildgebung denn schon wieder so lange her? Eigentlich ja, Der Radiologe widmete sich der Leberdiagnostik in Themenheften der Jahre 2004 und 2005.

Was hat sich seither getan? Zugegeben, die Pathologie der Leberläsionen ist grundsätzlich die gleiche geblieben, aber die Einsicht in die pathologischen Vorgänge gerade bei lebereigenen Tumoren hat sich vertieft. Neue pathologische Erkenntnisse lassen uns so z. B. das gelegentlich sehr komplexe und verwirrende Erscheinungsbild von Leberzelladenomen besser verstehen.

Die steigenden Anforderungen an die abdominelle Bildgebung bringen es mit sich, dass wie in anderen Organen auch in der Leber immer mehr „Läsionen“ entdeckt werden. Dabei genügt es nicht mehr, nur die Anzahl, Lokalisation und mögliche Differenzialdiagnose zu nennen, vielmehr ist abhängig von der Untersuchungsindikation und klinischen Situation der Patienten eine möglichst exakte Charakterisierung dieser Befunde anzustreben, um die betroffenen Patienten einer adäquaten Therapie zuführen zu können oder eine inadäquate Therapie zu vermeiden.

Dabei erlaubt uns das zunehmende Verständnis leber- und läsionspezifischer Stoffwechselvorgänge und deren Auswirkungen auf die Bildgebung gerade unter Verwendung moderner Kontrastmittel für Ultraschall und MRT, hepatische Herdbefunde zunehmend präziser zu differenzieren.
Es freut uns sehr, dass wir für das Ihnen nun vorliegende Themenheft namhafte Autoren mit ausgewiesener Expertise auf dem Gebiet der Leber gewinnen konnten, die Ihnen einen umfassendes Update typischer und untypischer Leberbefunde einschließlich pathoanatomischer Grundlagen und modernster Untersuchungstechnik mittels Ultraschall, CT, PET/CT und MRT geben.

Viel Vergnügen beim Lesen

Ihre

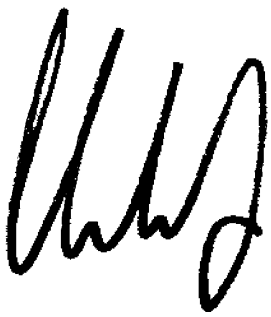

Prof. Dr. Thomas Helmberger

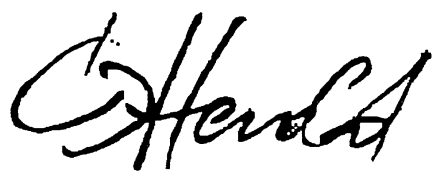

Prof. Dr. Christian Herold 